\title{
Surgical disconnection of a hypoglossal canal dural arteriovenous fistula demonstrating rapid progression of medulla oblongata disturbance: illustrative case
}

\author{
Yuichi Kawasaki, MD, ${ }^{1}$ Fumiaki Kanamori, MD, PhD, ${ }^{2}$ Tetsuya Tsukada, MD, PhD, ${ }^{1}$ Kazunori Shintai, MD, PhD, ${ }^{1}$ \\ Syuntaro Takasu, MD, PhD, ${ }^{1}$ and Yukio Seki, MD, PhD ${ }^{1}$ \\ 1Department of Neurosurgery, Japanese Red Cross Nagoya Daini Hospital, Nagoya, Aichi, Japan; and ${ }^{2}$ Department of Neurosurgery, Nagoya University Graduate School of
Medicine, Nagoya, Japan
}

\begin{abstract}
BACKGROUND Dural arteriovenous fistulas of the hypoglossal canal (HCDAVFs) with dominant drainage to perimedullary veins are extremely rare These patients are prone to develop slow and progressive myelopathy, however, their clinical course has not been fully elucidated. We report an unusual case of HCDAVF in which the patient demonstrated rapid progression of hemiplegia and respiratory insufficiency.

OBSERVATIONS An 82-year-old woman demonstrated motor weakness of the left extremities. T2-weighted magnetic resonance imaging showed a high intensity area in the right medulla oblongata and angiography revealed HCDAVF with dominant drainage to the anterior medullary vein through the anterior condylar vein. Within 3 days, her hemiparesis and respiratory function worsened, and she needed mechanical ventilation. Considering that venous congestion in the medulla oblongata could cause the symptoms, we immediately performed surgical obliteration of the anterior condylar vein. The disappearance of HCDAVF was confirmed by angiography and the patient was weaned from mechanical ventilation 3 days postoperatively. Her left hemiplegia gradually resolved and she was independent in daily life 8 months after the operation.
\end{abstract}

LESSONS HCDAVFs with dominant drainage to the perimedullary veins can demonstrate rapid progression of medulla oblongata disturbance. Early disconnection should be considered to provide an opportunity for substantial recovery.

https://thejns.org/doi/abs/10.3171/CASE21657

KEYWORDS hypoglossal canal dural arteriovenous fistula; rapid progression of the medulla oblongata disturbance; surgical obliteration

Dural arteriovenous fistulas of the hypoglossal canal (HCDAVFs) are a rare subtype of dural arteriovenous fistulas (DAVFs), which accounts for $3.6-4.2 \%$ of all intracranial DAVFs. ${ }^{1,2}$ The shunt point exists on the anterior condylar vein (ACV) or within the intraosseous structure around the hypoglossal canal. ${ }^{3-5}$ Furthermore, the ACV is the main venous drainage route that has connections with the anterior condylar confluence (ACC) outside the cranium and the epidural veins inside the cranium, such as the marginal sinus or basilar plexus. Depending on the drainage pattern, patients with HCDAVFs show various clinical presentation such as pulse-synchronous tinnitus, bruit, orbital symptoms, hypoglossal nerve palsy, myelopathy, and intracerebral hemorrhage. ${ }^{6}$

Among the subset of HCDAVFs classified according to the drainage pattern, cases with dominant venous drainage to perimedullary veins are even less frequent. Patients having HCDAVFs with this drainage pattern are prone to present slow progressive myelopathy, ${ }^{6}$ however, their clinical course has not been fully elucidated. Herein, we report a patient having HCDAVF with dominant venous drainage to the anterior medullary vein demonstrating rapid progression of hemiplegia and respiratory insufficiency, who was treated by surgical disconnection and showed good recovery.

ABBREVIATIONS ACC = anterior condylar confluence; $\mathrm{ACV}=$ anterior condylar vein; $\mathrm{DAVF}=$ dural arteriovenous fistula; $\mathrm{HCDAVF}=$ dural arteriovenous fistula of the hypoglossal canal; MRI = magnetic resonance imaging.

INCLUDE WHEN CITING Published February 7, 2022; DOI: 10.3171/CASE21657.

SUBMITTED November 20, 2021. December 20, 2021.

(C) 2022 The authors, CC BY-NC-ND 4.0 (http://creativecommons.org/licenses/by-nc-nd/4.0/). 

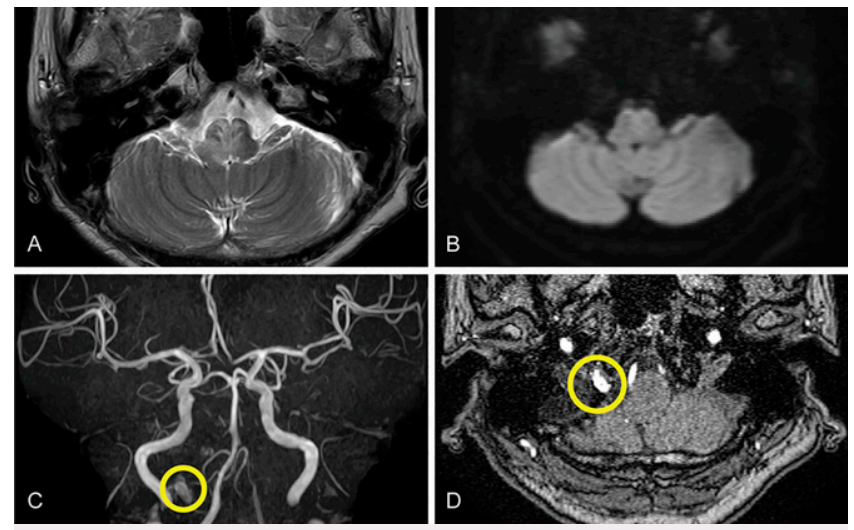

FIG. 1. Magnetic resonance images (MRI) on admission. T2-weighted MRI (A) showed a high intensity area mainly in the right medulla oblongata, but not in the diffusion-weighted image (B). The 3D reconstructed and raw images of magnetic resonance angiography showed a high intensity region (yellow circle) in the right hypoglossal canal (C and D). $3 \mathrm{D}=$ three dimensional.

\section{Illustrative Case}

An 82-year-old woman without any history of notable head trauma experienced weakness in the legs. Eight days after the onset, she presented to our department. At the time of admission, the patient could not walk unassisted, leaned to the left, and needed a wheelchair for movement. She showed hemiparesis with severe motor weakness of the left leg and mild weakness of the left arm. She also showed slight paresis of the right leg. Although neurological examinations of cranial nerves were normal, she claimed that she experienced difficulty with speech. The Babinski response was positive on both sides. T2weighted magnetic resonance imaging (MRI) identified a high intensity area mainly in the right medulla oblongata and the area was negative in diffusion-weighted imaging (Fig. $1 \mathrm{~A}$ and $\mathrm{B}$ ). Magnetic resonance angiography showed an unusual high intensity region in the right hypoglossal canal (Fig. 1C and D). Cervical MRI was also checked, but it showed only slight stenosis. Dysphagia was observed the next day. Two days after admission, her motor weakness progressed, and she was unable to move left extremities. In addition, she developed hoarseness and urinary incontinence. Angiography revealed HCDAVF, which was fed by the hypoglossal branch of the right ascending pharyngeal artery, shunted just around the hypoglossal canal, and drained into the anterior medullary vein through the ACV (Fig. 2). On the day after angiography by early morning, she found it difficult to breathe spontaneously and needed management with tracheal intubation, and mechanical ventilation. Considering that venous congestion in the medulla oblongata could have caused rapid progression of hemiplegia and respiratory insufficiency, we performed surgical disconnection of the HCDAVF via the suboccipital transcondylar approach on the same day as soon as possible (Video 1). In the operative field, the diameter of the ACV was large and appeared reddish (Fig. 3A). After confirmation of arteriovenous shunt flow using Doppler ultrasound or indocyanine green video angiography, we obliterated the ACV just after the outlet of the hypoglossal canal by Sugita Titanium II clip No. 89

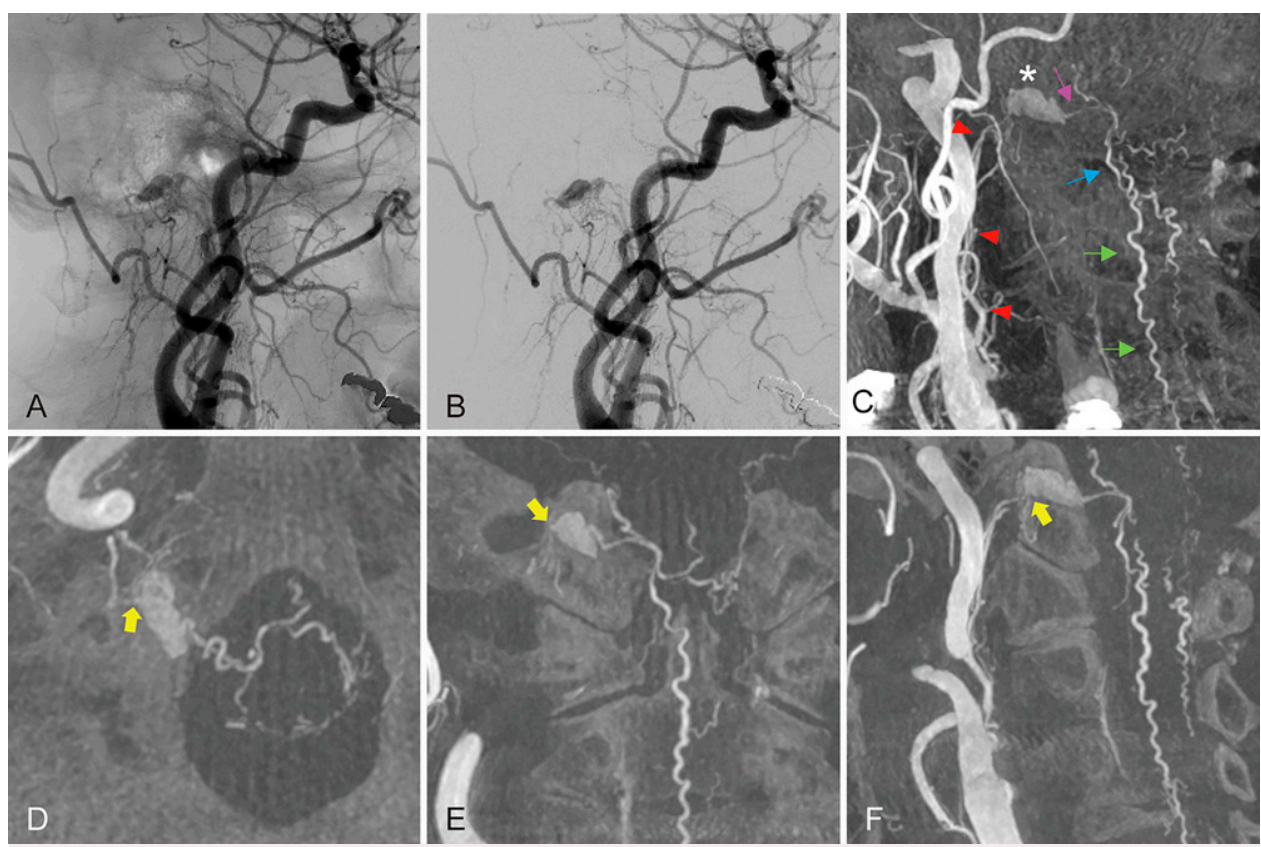

FIG. 2. Preoperative images of the right common carotid artery angiography. The right common carotid artery angiogram in lateral view (A) and digital subtraction angiogram (B) revealed a dural arteriovenous fistula of the hypoglossal canal. 3D rotation angiogram demonstrated the overall structure of the dural arteriovenous fistula of the hypoglossal canal (C). The fistula was fed by the hypoglossal branch of the right ascending pharyngeal artery (red arrowhead). The arteriovenous shunt flow first drained into the anterior condylar vein (asterisk), next to the bridging vein (pink arrow), and finally flowed into the anterior medullary vein (blue arrow) that led to the anterior spinal vein (green arrow). Slab maximum intensity projection images (D, axial; E, coronal; $\mathbf{F}$, sagittal) showed the shunt point of the dural arteriovenous fistula just around the hypoglossal canal (yellow arrow) and dilated perimedullary veins. $3 \mathrm{D}=$ three dimensional. 

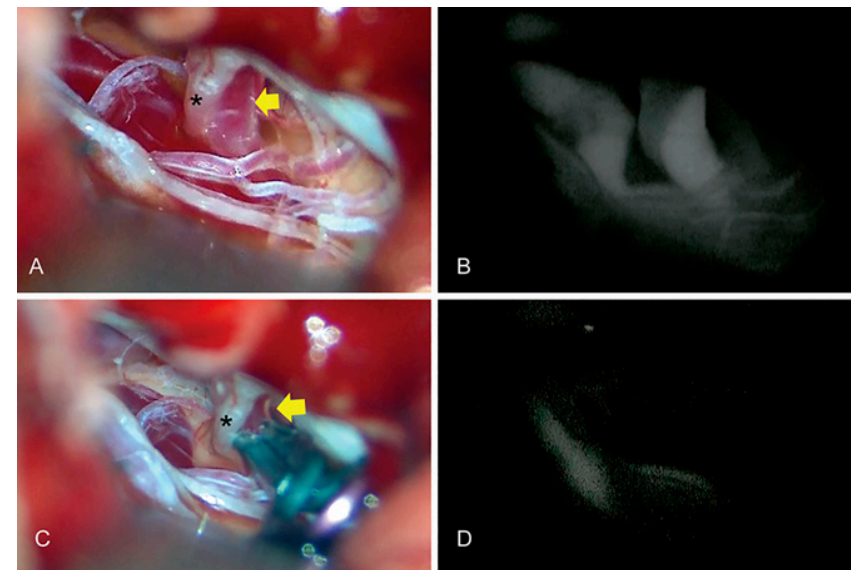

FIG. 3. Intraoperative images of the surgical disconnection of hypoglossal canal dural arteriovenous fistula. The anterior condylar vein (yellow arrow) just beside the hypoglossal nerve (asterisk) was large and seemed reddish (A). The arteriovenous shut flow was confirmed by indocyanine green video angiography $(\mathbf{B})$. The anterior condylar vein was obliterated by Sugita Titanium II clip No. 89 (C), and indocyanine green video angiography showed the disappearance of arteriovenous shunt flow (D).

(Mizuho Co., Ltd) (Fig. 3B-D). Postoperative angiography revealed the disappearance of the HCDAVF (Fig. 4A). Three days after the operation, although a slight left vocal cord paresis remained, the patient was able to breathe by herself and was weaned from mechanical ventilation. Hypoglossal nerve palsy was not observed. Her left hemiparesis and dysphagia recovered gradually and by 1 month after the operation, she could maintain a standing position and consume meals. The high intensity area in the medulla oblongata in T2-weighted MRI decreased and the high intensity region of the right hypoglossal canal in magnetic resonance angiography disappeared (Fig. 4B and C). She continued rehabilitation for 8 months. Despite a slight motor weakness in the left extremity, she became independent in daily life.
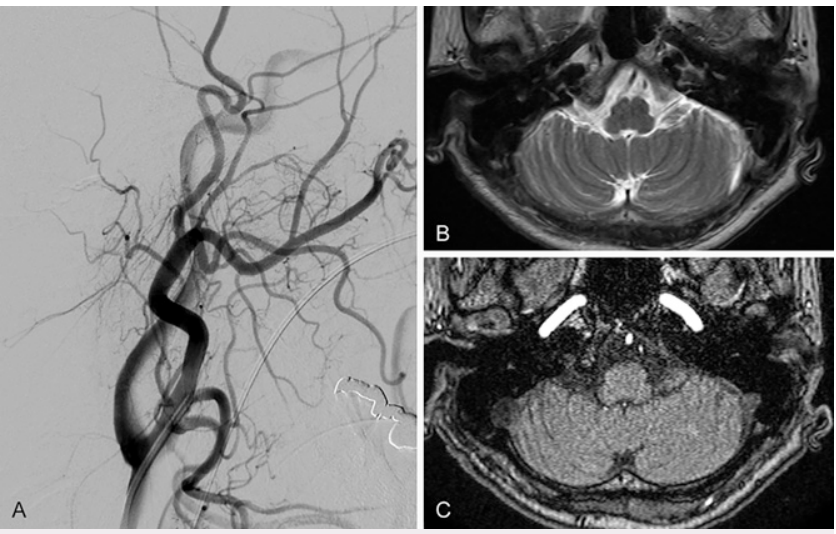

FIG. 4. Postoperative images of angiography and magnetic resonance imaging (MRI). The right common carotid artery angiogram in the lateral view (A) shows the disappearance of hypoglossal canal dural arteriovenous fistula. MRI 8 months after the operation did not show the abnormal high intensity region in T2-weighted MRI (B) and magnetic resonance angiography $(\mathbf{C})$.

VIDEO 1. Short operative video of the present case: surgical disconnection of the HCDAVF. Click here to view.

\section{Discussion}

\section{Observations}

HCDAVFs with dominant drainage to the perimedullary veins are exceedingly rare. In the largest systematic review including 120 cases of HCDAVFs, ${ }^{6}$ only 16 cases had perimedullary drainage..$^{2,3,7-18}$ Cases of HCDAVFs with perimedullary drainage usually manifest with slow progressive symptoms related to cervical myelopathy. ${ }^{6}$ The unique clinical course of the present case was rapid progression of hemiplegia and respiratory insufficiency due to venous congestion in the medulla oblongata. To date, rapid progression of medulla oblongata disturbance has been reported in only two cases (Table 1). ${ }^{13,14}$ Including the present case, all three cases

TABLE 1. Cases of HCDAVFs demonstrating rapid progression of medulla oblongata disturbance

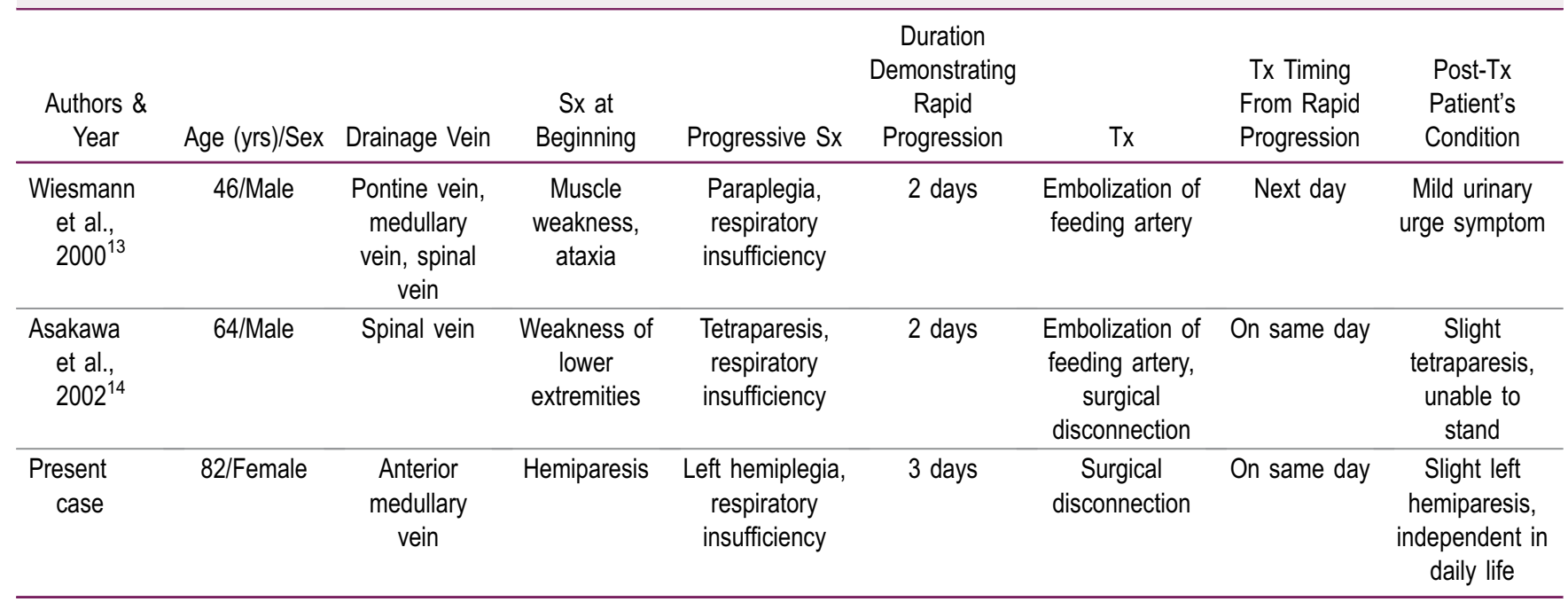

Sx = symptoms; $T x=$ treatment. 
required hospitalization with paresis; however, they showed rapid progression of plegia and respiratory insufficiency within three days and needed mechanical ventilation. Although the cause of rapid progression remains unknown, we speculate that obliteration of originally functioned other drainage routes, such as the ACC, and could have triggered rapid progression; the increased drainage flow in perimedullary veins would have worsened the venous congestion in the medulla oblongata. Regarding therapeutic management, these three cases received surgical or endovascular treatments within 1 day after rapid progression. Their clinical course after treatment were favorable; they were weaned from ventilation and their plegia improved substantially. The recovery might have been achieved by early HCDAVF disconnection that was performed before ischemic changes due to venous congestion became irreversible. Even in HCDAVF cases with dominant drainage to perimedullary veins who demonstrated rapid neurological deterioration, although the interval for venous infarction may vary from case to case, early disconnection of the arteriovenous shunt should be performed to obtain a good clinical outcome.

In the present case, HCDAVF was fed by the hypoglossal branch of the ascending pharyngeal artery, shunted just around the hypoglossal canal, and drained into the anterior medullary vein through the ACV. Although endovascular treatment is a major treatment method for HCDAVFs, ${ }^{6}$ surgical obliteration is also useful in the drainage conditions presented here. Transvenous coil embolization of the ACV has a risk of hypoglossal nerve palsy due to the mass effect from the coil on the hypoglossal nerve. ${ }^{1,10,19}$ To prevent this complication, over packing of the ACV should be avoided or superselective coil embolization of the shunted venous pouch is required. Besides, transarterial embolization using Onyx or N-butyl cyanoacrylate also has a significant risk of hypoglossal nerve palsy because the hypoglossal branch (known as neuromeningeal branch) of the ascending pharyngeal artery, which also supplies the vasa nervorum of the hypoglossal nerve, was the feeder of this HCDAVF case. ${ }^{20,21}$ In contrast, surgical disconnection of the arteriovenous shunt had seemed to require the simple procedure of obliterating the $A C V$, since the ACV was the single drainer just after the arteriovenous fistula. Based on these angiographical characteristics and surgical anatomy, we assumed that the surgical difficulty was not high and the risk of hypoglossal nerve injury in surgery was relatively low. Thus, we selected surgical obliteration of the ACV via the transcondylar approach and obtained a favorable outcome.

The majority of HCDAVFs develop with pulsatile tinnitus or ocular symptoms. Pulse-synchronous tinnitus is often observed in the HCDAVF patients with dominant anterograde venous drainage to the internal jugular vein and/or vertebral venous plexus, and orbital symptoms (chemosis, proptosis, and diplopia) is observed in those with dominant retrograde drainage to the cavernous sinus and/or orbital veins. On these drainage conditions, transvenous embolization is a highly safe and effective treatment. ${ }^{6}$ Compared to these major symptoms or treatment strategy of HCDAVFs, the present case demonstrated quite different symptoms and needed other management. However, it should be noted that HCDAVF can cause rapid progression of medulla oblongata disturbance and surgical disconnection could be the option of treatment.

\section{Lessons}

HCDAVFs with dominant drainage to perimedullary veins can present as rapid progression of plegia and respiratory insufficiency due to venous congestion of medulla oblongata. Early disconnection of the arteriovenous shunt should be performed to provide an opportunity for a remarkable recovery. In addition, for the HCDAVF cases with the ACV as a single drainer, surgical intervention can be a good option. As a limitation, the cases with unfavorable outcomes may be less likely to be reported, a large cohort study is needed for validation.

\section{References}

1. Manabe S, Satoh K, Matsubara S, Satomi J, Hanaoka M, Nagahiro S. Characteristics, diagnosis and treatment of hypoglossal canal dural arteriovenous fistula: report of nine cases. Neuroradiology. 2008;50(8):715-721.

2. Choi JW, Kim BM, Kim DJ, et al. Hypoglossal canal dural arteriovenous fistula: incidence and the relationship between symptoms and drainage pattern. J Neurosurg. 2013;119(4):955-960.

3. Ernst RJ, Gaskill-Shipley M, Tomsick TA, Hall LC, Tew JM Jr, Yeh HS. Cervical myelopathy associated with intracranial dural arteriovenous fistula: MR findings before and after treatment. AJNR Am J Neuroradiol. 1997;18(7):1330-1334.

4. Jung C, Kwon BJ, Kwon OK, et al. Intraosseous cranial dural arteriovenous fistula treated with transvenous embolization. AJNR Am J Neuroradiol. 2009;30(6):1173-1177.

5. Mizutani K, Akiyama T, Minami Y, et al. Intraosseous venous structures adjacent to the jugular tubercle associated with an anterior condylar dural arteriovenous fistula. Neuroradiology. 2018;60(5):487-496.

6. Spittau B, Millán DS, El-Sherifi S, et al. Dural arteriovenous fistulas of the hypoglossal canal: systematic review on imaging anatomy, clinical findings, and endovascular management. J Neurosurg. 2015;122(4):883-903.

7. Barnwell SL, Halbach VV, Dowd CF, Higashida RT, Hieshima GB. Dural arteriovenous fistulas involving the inferior petrosal sinus: angiographic findings in six patients. AJNR Am J Neuroradiol. 1990;11(3):511-516.

8. Rodesch G, Comoy J, Hurth M, Lasjaunias P. Jugular foramen arteriovenous shunt with subarachnoid hemorrhage. Skull Base Surg. 1991;1(2):132-136.

9. Mascalchi M, Scazzeri F, Prosetti D, Ferrito G, Salvi F, Quilici N. Dural arteriovenous fistula at the craniocervical junction with perimedullary venous drainage. AJNR Am J Neuroradiol. 1996;17(6):1137-1141.

10. McDougall CG, Halbach VV, Dowd CF, Higashida RT, Larsen DW, Hieshima GB. Dural arteriovenous fistulas of the marginal sinus. AJNR Am J Neuroradiol. 1997;18(8):1565-1572.

11. Hähnel S, Jansen $O$, Geletneky K. MR appearance of an intracranial dural arteriovenous fistula leading to cervical myelopathy. Neurology. 1998;51(4):1131-1135.

12. Hurst RW, Bagley LJ, Scanlon M, Flamm ES. Dural arteriovenous fistulas of the craniocervical junction. Skull Base Surg. 1999;9(1):1-7.

13. Wiesmann M, Padovan CS, Pfister HW, Yousry TA. Intracranial dural arteriovenous fistula with spinal medullary venous drainage. Eur Radiol. 2000;10(10):1606-1609.

14. Asakawa H, Yanaka K, Fujita K, et al. Intracranial dural arteriovenous fistula showing diffuse MR enhancement of the spinal cord: case report and review of the literature. Surg Neurol. 2002;58(34):251-257.

15. Chng SM, Sitoh YY, Hui F. Intracranial dural arteriovenous fistula presenting with tetraparesis due to cervicomedullary junction compression. A case report. Interv Neuroradiol. 2004;10(4):347-351.

16. Tanoue S, Goto K, Oota S. Endovascular treatment for dural arteriovenous fistula of the anterior condylar vein with unusual venous 
drainage: report of two cases. AJNR Am J Neuroradiol. 2005;26(8): 1955-1959.

17. Byun JS, Hwang SN, Park SW, Nam TK. Dural arteriovenous fistula of jugular foramen with subarachnoid hemorrhage : selective transarterial embolization. J Korean Neurosurg Soc. 2009;45(3): 199-202.

18. Kai Y, Hamada J, Morioka M, Ushio Y, Fujioka S. Foramen magnum dural arteriovenous fistulae with repeated subarachnoid haemorrhage. Interv Neuroradiol. 1998;4(2):171-176.

19. Miyachi S, Ohshima T, Izumi T, Kojima T, Yoshida J. Dural arteriovenous fistula at the anterior condylar confluence. Interv Neuroradiol. 2008;14(3):303-311.

20. Hacein-Bey L, Daniels DL, Ulmer JL, et al. The ascending pharyngeal artery: branches, anastomoses, and clinical significance. AJNR Am J Neuroradiol. 2002;23(7):1246-1256.

21. Hellstern V, Aguilar-Pérez M, Schob S, et al. Endovascular treatment of dural arteriovenous fistulas of the anterior or posterior condylar vein : a cadaveric and clinical study and literature review. Clin Neuroradiol. 2019;29(2):341-349.

\section{Disclosures}

The authors report no conflict of interest concerning the materials or methods used in this study or the findings specified in this paper.

\section{Author Contributions}

Conception and design: Takasu, Kawasaki, Kanamori. Acquisition of data: Takasu, Kawasaki, Tsukada, Shintai. Analysis and interpretation of data: Takasu, Kawasaki, Tsukada. Drafting the article: Takasu, Kawasaki, Kanamori. Critically revising the article: Takasu, Kanamori. Reviewed submitted version of manuscript: Takasu, Tsukada. Approved the final version of the manuscript on behalf of all authors: Takasu. Statistical analysis: Takasu. Administrative/technical/material support: Takasu. Study supervision: Takasu, Seki.

\section{Supplemental Information \\ Video}

Video 1. https://vimeo.com/660945892.

\section{Correspondence}

Syuntaro Takasu: Japanese Red Cross Nagoya Daini Hospital, Aichi, Japan. syuntaro@xj.commufa.jp. 